\title{
Effects of neonatal enteral glutamine supplementation on cognitive, motor and behavioural outcomes in very preterm and/or very low birth weight children at school age
}

\author{
Jorrit F. de Kieviet ${ }^{1 *}$, Jaap Oosterlaan ${ }^{1}$, Annelies van Zwol $^{2}$, Guenther Boehm ${ }^{3,4}$, Harrie N. Lafeber ${ }^{2}$ \\ and Ruurd M. van Elburg ${ }^{2}$ \\ ${ }^{1} V U$ University Amsterdam, Department of Clinical Neuropsychology, Amsterdam, The Netherlands \\ ${ }^{2}$ VU University Medical Center, Department of Pediatrics, Amsterdam, The Netherlands \\ ${ }^{3}$ Danone Research Friedrichsdorf, Friedrichsdorf, Germany \\ ${ }^{4}$ Sophia Children's Hospital, Erasmus University, Department of Pediatrics, Rotterdam, The Netherlands
}

(Submitted 1 September 2011 - Final revision received 16 January 2012 - Accepted 16 January 2012 - First published online 7 February 2012)

\section{Abstract}

In very preterm ( $<32$ weeks of gestation) and/or very low birth weight (VLBW, $<1500 \mathrm{~g}$ birth weight) children, serious neonatal infections are among the main causes of poor developmental outcomes later in childhood. The amino acid glutamine has been shown to reduce the incidence of serious neonatal infections in very preterm and/or VLBW children, while developmental effects beyond 24 months are unknown. We determined the cognitive, motor and behavioural outcomes at school age of a cohort of sixty-four very preterm and/or VLBW children (aged 7.5 (SD 0.4 ) years) who participated in a randomised placebo-controlled trial using enteral glutamine between day 3 and day 30 of life. Cognitive and motor outcomes were studied using the Wechsler Intelligence Scale for Children-III, the Movement Assessment Battery for Children (MABC), the Attention Network Test and a visual working memory task. Behavioural outcomes were evaluated using parent- and teacher-rated questionnaires. Intelligence quotient, processing speed, attentional functioning, working memory and parent- and teacher-rated behavioural outcomes were not different between children treated with glutamine or placebo; only visuomotor abilities as measured by the Ball Skills scale of the MABC $(P=0 \cdot 002 ; d=0 \cdot 67)$ were poorer in the glutamine group. This effect persisted after taking into account the beneficial effects of lower serious neonatal infections rates in children treated with glutamine $(P=0 \cdot 005)$. In conclusion, glutamine supplementation between day 3 and day 30 of life had neither beneficial nor detrimental effects on long-term cognitive, motor and behavioural outcomes of very preterm and/or VLBW children at school age, although visuomotor abilities were poorer in children that received glutamine.

\section{Key words: Neonatal enteral glutamine: Preterm children: Very low birth weight children: Development: Behavioural} outcomes

With advances in neonatal intensive care, the survival of very preterm (gestational age $(\mathrm{GA})<32$ weeks) and/or very low birth weight (VLBW; birth weight < 1500 g) children has improved considerably. However, these children are at risk for poor motor, cognitive and behavioural outcomes later in childhood $^{(1,2)}$ due to a variety of risk factors associated with preterm birth. Risk factors include neonatal infections and inflammatory responses, both contributing to early brain injury $^{(3)}$.

Over the past decade, the potential protective effects of supplementation of the amino acid glutamine in very preterm and/or VLBW children have been extensively studied, including the modulation of inflammatory response and stimulation of immunity ${ }^{(4)}$. Experimental studies have shown that glutamine plays an important role in maintaining the functional integrity of the gut ${ }^{(5,6)}$, which in turn leads to decreased bacterial translocation and systemic spread of bacteria ${ }^{(7-9)}$, and consequently may lead to decreased infectious morbidity. Indeed, a few studies found that glutamine-enriched enteral nutrition between day 3 and day 30 of life decreased the number of serious neonatal infections in very preterm and/ or VLBW children ${ }^{(10,11)}$, although other studies failed to replicate the beneficial effects of glutamine ${ }^{(12)}$. A lower number of serious neonatal infections may potentially be beneficial for long-term motor, cognitive and behavioural outcomes in very preterm and/or VLBW children, as evidence from several

Abbreviations: GA, gestational age; MABC, Movement Assessment Battery for Children; PDBD, parent-rated Disruptive Behaviour Disorders questionnaire; SES, socio-economic status; TDBD, teacher version of the Disruptive Behaviour Disorders questionnaire; TRF, Teacher's Report Form; VLBW, very low birth weight.

*Corresponding author: J. F. de Kieviet, fax +31 205988971, email jf.de.kieviet@vu.nl 
studies emphasises the negative effects of neonatal infections on long-term neurodevelopment ${ }^{(13-16)}$. One study found no beneficial or adverse effects of enteral glutamine supplementation on mental or motor outcomes at 2 years of age as measured by the Bayley Scales of Infant Development, second edition $^{(17)}$, while another study found that long-term enteral glutamine supplementation may lead to significant improvements in growth measures ${ }^{(18)}$. However, until now it remains unclear whether a lower incidence of neonatal infections and improved growth in very preterm and/or VLBW children following glutamine supplementation may be beneficial for long-term motor, cognitive and behavioural development.

The aim of the present study was to determine the long-term effects of enteral glutamine, supplemented in a randomised placebo-controlled trial between day 3 and day 30 of life, on the cognitive, motor and behavioural outcomes of very preterm and/or VLBW children at school age.

\section{Methods}

The initial sample for the present study consisted of 102 very preterm $(<32$ weeks $)$ and/or VLBW $(<1500 \mathrm{~g})$ infants participating in a randomised placebo-controlled trial on glutamine supplementation. In the present study, infants received enteral glutamine supplementation $(0.3 \mathrm{~g} / \mathrm{kg}$ per $\mathrm{d})$ or an isonitrogenous placebo supplementation (alanine) between day 3 and day 30 of life. All very preterm and/or VLBW infants admitted to the level III neonatal intensive care unit (NICU) of the VU University Medical Center Amsterdam between September 2001 and July 2003 were eligible for inclusion. Descriptions of baseline characteristics and techniques used to determine incidence of serious neonatal infections, intraventricular haemorrhage, periventricular leucomalacia and bronchopulmonary dysplasia have been previously reported ${ }^{(11)}$. Of the 102 infants included in the study, eighty-nine infants were alive at 1 year of age, and seventy-four were still participating at 6 years of age.

At 7-8 years of age, parents of all seventy-four children were contacted and invited to participate in the present study, of which sixty-eight (92\%) showed up, and sixty-four (94\%) successfully completed all tasks at the mean age of 7.5 (SD 0.4) years. The remaining four children had serious motor ( $n$ 2), hearing ( $n$ 1) or vision ( $n 1)$ difficulties, crucially interfering with task execution. The final sample consisted of thirty children that had received glutamine (glutamine group) and thirty-four children that had received placebo (control group). From the original dataset, the presence of serious neonatal infections and other clinical complications were extracted. Serious neonatal infections included sepsis, meningitis, pyelonephritis, pneumonia and arthritis, accompanied by positive microbial cultures of blood, cerebrospinal fluid, urine, tracheal aspirates and synovial fluid, respectively, as previously described in more detail ${ }^{(11)}$. Socio-economic status (SES) was determined by classifying the highest level of education in a household with a number ranging from 0 to 4 . A higher number indicated a higher level of education and a corresponding higher SES. Characteristics of both groups are shown in Table 1.

\section{Procedure}

The present study was conducted according to the guidelines laid down in the Declaration of Helsinki and all procedures involving human subjects were approved by the medical ethical committee of the VU University Medical Center. Written informed consent was obtained from all subjects. Cognitive and motor assessment took place at the VU University Amsterdam by qualified and trained testers using a completely standardised instruction protocol. Both parents and each child's teacher were asked to fill in questionnaires addressing behavioural problems at home and at school, respectively. Parents filled in the questionnaires in the presence of an interviewer.

\section{Cognitive and motor measures}

The main aspects of cognitive and motor functioning were assessed, including intellectual development, working memory, motor development, aspects of attentional functioning and processing speed. Intellectual development was measured by a short form of the Wechsler Intelligence Scale for Children-III (WISC-III ${ }^{(19)}$ ), including the subtests

Table 1. Sample characteristics

(Mean values and standard deviations; numbers of participants and percentages; medians and ranges)

\begin{tabular}{|c|c|c|c|c|c|}
\hline \multirow[t]{2}{*}{ Treatment group... } & \multicolumn{2}{|c|}{$\begin{array}{c}\text { Placebo } \\
(n 34)\end{array}$} & \multicolumn{2}{|c|}{$\begin{array}{l}\text { Glutamine } \\
\quad(n 30)\end{array}$} & \multirow[b]{2}{*}{$P^{*}$} \\
\hline & $n$ & $\%$ & $n$ & $\%$ & \\
\hline Age at assessment (years) & \multirow{2}{*}{\multicolumn{2}{|c|}{7.5}} & \multirow{2}{*}{\multicolumn{2}{|c|}{$7 \cdot 5$}} & 0.45 \\
\hline Mean & & & & & \\
\hline SD & \multicolumn{2}{|c|}{0.4} & \multicolumn{2}{|c|}{0.4} & \\
\hline Birth weight (g) & & & & & 0.28 \\
\hline Mean & \multicolumn{2}{|c|}{1204} & \multicolumn{2}{|c|}{1301} & \\
\hline SD & \multicolumn{2}{|c|}{334} & \multicolumn{2}{|c|}{380} & \\
\hline Gestational age (weeks) & \multirow{2}{*}{\multicolumn{2}{|c|}{$29 \cdot 0$}} & & & 0.04 \\
\hline Mean & & & \multicolumn{2}{|c|}{$29 \cdot 7$} & \\
\hline SD & \multicolumn{2}{|c|}{1.6} & \multicolumn{2}{|c|}{1.6} & \\
\hline Head circumference $(\mathrm{cm})$ & \multirow{2}{*}{\multicolumn{2}{|c|}{26.7}} & & & 0.12 \\
\hline Mean & & & \multicolumn{2}{|c|}{$27 \cdot 6$} & \\
\hline SD & \multicolumn{2}{|c|}{2.5} & \multicolumn{2}{|c|}{$2 \cdot 0$} & \\
\hline Socio-economic status & \multirow{2}{*}{\multicolumn{2}{|c|}{$3 \cdot 0$}} & & & 0.04 \\
\hline Mean & & & \multicolumn{2}{|c|}{$3 \cdot 2$} & \\
\hline SD & \multicolumn{2}{|c|}{0.7} & \multicolumn{2}{|c|}{0.7} & \\
\hline Male sex & 17 & 50 & 15 & 50 & \\
\hline \multicolumn{6}{|l|}{ Birth weight (g) } \\
\hline Median & \multirow{2}{*}{\multicolumn{2}{|c|}{$\begin{array}{c}1175 \\
690-1795\end{array}$}} & \multicolumn{2}{|c|}{1268} & \\
\hline Range & & & 560 & 325 & \\
\hline \multicolumn{6}{|l|}{ Gestational age (weeks) } \\
\hline Median & \multicolumn{2}{|c|}{$29 \cdot 0$} & & & \\
\hline Range & $25 \cdot 7$ & $31 \cdot 7$ & 25.4 & $31 \cdot 7$ & \\
\hline Birth weight $<10$ th percentile & 8 & 24 & 8 & 27 & 0.77 \\
\hline Maternal HELLP syndrome & 6 & 18 & 4 & 13 & 0.64 \\
\hline Prenatal corticosteroids & 31 & 91 & 22 & 73 & 0.06 \\
\hline BPD & 11 & 32 & 7 & 23 & 0.42 \\
\hline IVH grade I/II & 6 & 18 & 6 & 20 & 0.81 \\
\hline IVH grade III/IV & 0 & 0 & 1 & 3 & 0.28 \\
\hline PVL & 2 & 6 & 1 & 3 & 0.63 \\
\hline Apgar score after $5 \min <6$ & 2 & 6 & 3 & 10 & 0.54 \\
\hline Caesarean section & 19 & 56 & 17 & 57 & 0.95 \\
\hline One or more serious infections & 27 & 79 & 14 & 47 & $<0.01$ \\
\hline
\end{tabular}

HELLP, haemolysis elevated liver enzymes and low platelets; BPD, bronchopulmonary dysplasia; IVH, intraventricular haemorrhage; PVL, periventricular leucomalacia.

${ }^{*} \chi^{2}$ and $t$ tests. 
Vocabulary and Block Design. Both subtests correlate strongly $(r>0.90)$ with full-scale intelligence quotient ${ }^{(20)}$. Scores on this test were normalised with a mean of 100 (SD 15). Two aspects of working memory abilities were assessed: verbal working memory and visual working memory. Verbal working memory abilities were measured using the Digit Span subtest of the WISC-III. In this subtest, children had to verbally reproduce dictated series of digits increasing in length, both in a forward and a backward condition. Maximal spans of reproduced digits for both the forward as well as the backward condition were included as dependent variables in the analysis. Visual working memory abilities were measured using an adapted version of a task developed by Nutley et $a l^{(21)}$. In this task, children had to reproduce sequences of circles appearing in a $4 \times 4$ grid on a touch screen. Difficulty level was increased during the course of the task by increasing the span and by manipulating the position of the stimuli. Two trials were administrated for each difficulty level, and the task was terminated when the child failed to accomplish both trials at a certain difficulty. Maximal difficulty levels with forward reproduction and backward reproduction were included as dependent variables in the analysis. Motor development was assessed using the Movement Assessment Battery for Children $(\mathrm{MABC})^{(22)}$, and outcomes on the scales Manual Dexterity, Balance Skills and Ball Skills were included in the analysis. Scores on the scales of the MABC were normalised using T-scores with a mean of 50 (SD 10). Orienting, executive and alerting attention was assessed using an adapted version of the Attention Network Test ${ }^{(23)}$ suitable for use with young children. In this task, children had to respond as accurately and quickly as possible to the appearance of a target on the left side or the right side of the screen by pressing a button corresponding to the location at which the target appeared. There were four types of trials. Neutral trials contained a neutral cue in the middle of the screen which preceded the target. Orienting trials contained a directional cue in the middle of the screen pointing to the position of the target which subsequently followed. Executive trials contained directional cues incongruent with the position of the target. Alerting trials contained no cue at all and the target was presented instantaneously. The four trial types were randomly presented in four blocks of forty-eight trials. Measures of orienting, executive and alerting attention were obtained by subtracting the mean response time on the orienting, executive and alerting trials from the mean response time on neutral trials, respectively. In this way, measures of orienting, alerting and executive attention are controlled for differences in information-processing capacities between children. The gain in response time in an orienting trial as compared with a neutral trial was used as a measure of the ability to aim attention (orienting attention). More gain in response time corresponds to better orienting attention. The loss in response time in an executive trial as compared with a neutral trial was used as a measure of the ability to actively ignore irrelevant information (executive attention). Less loss in response time corresponds to better executive attention. The loss in response time in an alerting trial as compared with a neutral trial was used as a measure of alertness (alerting attention). Less loss in response time corresponds to better alerting attention. Finally, the combined effects of cognitive and motor speed were assessed by mean response time in neutral trials and included as a measure of overall processing speed in the analysis.

\section{Behavioural measures}

Parents rated their children's behavioural difficulties using the Child Behaviour Checklist $(\mathrm{CBCL})^{(24)}$ and the parent-rated Disruptive Behaviour Disorders questionnaire (PDBD) ${ }^{(25)}$. Teachers rated their pupil's behavioural difficulties using the Teacher's Report Form (TRF) ${ }^{(26)}$ and teacher version of the Disruptive Behaviour Disorders questionnaire (TDBD) ${ }^{(25)}$ The CBCL and TRF encompass three broad-band scales assessing total, externalising and internalising behaviour problems, and the PDBD and TDBD comprise two scales addressing inattention and hyperactivity problems. Scores on all scales were depicted as normalised T-scores with a mean of 50 (SD 10); higher scores indicated greater severity of problems. All questionnaires are widely used and have excellent psychometric properties.

\section{Statistical analyses}

All analyses were performed using SPSS 17.0 (SPSS, Inc.), and raw continuous data were successfully standardised and normalised by applying a Van der Waerden transformation. Pearson and point biserial correlations were used to explore the effects of serious neonatal infections, SES and GA on cognitive, motor and behavioural outcomes at school age. To study the effects of glutamine supplementation, univariate ANOVA with intervention as a between-subject factor and SES and GA as covariates was conducted on the dependent measures of the cognitive and motor tasks and parent- and teacher-rated questionnaires. To adjust for possible beneficial indirect effects of glutamine on the outcome measures by decreasing the number of serious neonatal infections, the analysis was repeated with serious neonatal infections as a second between-subjects factor. Standardised group differences were quantified in terms of effect sizes (Cohen's $d)^{(27)}$, which is an effect size defined by the difference between two group means divided by the pooled SD, enabling the opportunity to indicate the size of an effect independent of group size. Cohen's $d$ guidelines were followed to indicate the strength of the group differences, with values of $0 \cdot 20,0 \cdot 50$ and $0 \cdot 80$ referring to small, medium and large effects, respectively ${ }^{(27)}$ To minimise the possibility of a type 2 error, which may erroneously lead to the conclusion that no detrimental or beneficial effects of glutamine-enriched feeding exist on long-term outcomes, $\alpha$ was not corrected for multiple comparisons and set at $0 \cdot 05$.

\section{Results}

\section{Sample characteristics}

Clinical characteristics of the glutamine and placebo groups are shown in Table 1 . The two groups did not differ on the 
majority of clinical characteristics, indicating that there were no differences in illness severity between the groups. However, SES and GA were higher for the glutamine group as compared with the placebo group. In addition, as was found in a previous study ${ }^{(11)}$, the rate of serious neonatal infections was lower in the glutamine group than in the placebo group $(P=0.006)$

\section{Correlational analyses}

Higher SES was associated with higher full-scale intelligence quotient scores $(r 0.26 ; P=0.002)$, and a shorter GA was associated with higher ratings on the TDBD Hyperactivity scale $(r-0.34 ; P=0.006)$. Furthermore, the presence of serious neonatal infections was positively associated with multiple scales of parent- and teacher-rated questionnaires, including TRF Total Problems ( $r 0 \cdot 25 ; P=0 \cdot 04)$, TRF Externalising ( $r$ 0.25;P=0.04), PDBD Inattention $(r 0.25 ; P=0.04)$, TDBD Inattention $(r 0 \cdot 27 ; P=0 \cdot 03)$ and TDBD Hyperactivity ( $r 0.27 ; P=0.03$ ), indicating that the presence of infections is associated with higher ratings of behavioural problems and symptoms of attention deficit hyperactivity disorder in particular.

\section{Cognitive, motor and behavioural outcomes}

Cognitive, motor and behavioural outcomes are shown in Table 2. After adjusting for SES, GA, and including the presence of serious neonatal infections as a second betweensubjects factor, the glutamine group showed poorer ball skills ( $P=0.002$; Cohen's $d=0 \cdot 67$ ), indicating that ball skills were more impaired in the glutamine group as compared with the placebo group. Furthermore, the glutamine group showed poorer executive attention as compared with the placebo group, although this effect was of marginal significance ( $P=0.06 ; d=0.49$ ). When only adjusting for SES and GA, poorer ball skills for the glutamine group remained significant $(P=0.005)$, indicating that a reduced presence of serious neonatal infections following glutamine supplementation could not compensate for the differences in ball skills between the glutamine and placebo groups. No significant differences were found between the glutamine and placebo groups for any of the remaining cognitive and motor measures.

\section{Behavioural outcomes}

Behavioural outcomes as measured by parent- and teacherrated questionnaires did not differ significantly between the

Table 2. Cognitive, motor and behavioural functioning for the placebo and glutamine groups

(Mean values and standard deviations)

\begin{tabular}{|c|c|c|c|c|c|c|c|}
\hline \multirow[t]{2}{*}{ Treatment group... } & \multicolumn{2}{|c|}{ Placebo ( $n$ 34) } & \multicolumn{2}{|c|}{ Glutamine ( $n$ 30) } & \multirow{2}{*}{$\begin{array}{l}\text { Effect size: } \\
\text { Cohen's } d\end{array}$} & \multirow{2}{*}{$\begin{array}{l}\text { Adjusted for GA, } \\
\text { SES, infections: } P\end{array}$} & \multirow{2}{*}{$\begin{array}{c}\text { Adjusted for GA, } \\
\text { SES: } P\end{array}$} \\
\hline & Mean & SD & Mean & SD & & & \\
\hline \multicolumn{8}{|l|}{ Cognitive functioning } \\
\hline WISC-III estimated FSIQ* & $93 \cdot 6$ & $17 \cdot 2$ & $102 \cdot 0$ & $17 \cdot 7$ & 0.48 & 0.23 & 0.12 \\
\hline \multicolumn{8}{|l|}{ Motor development } \\
\hline MABC Balance Skills* & $53 \cdot 1$ & 14.9 & 54.4 & $19 \cdot 8$ & 0.03 & 0.22 & 0.40 \\
\hline MABC Ball Skills* & $57 \cdot 4$ & $13 \cdot 0$ & $66 \cdot 0$ & $13 \cdot 9$ & 0.67 & 0.002 & 0.005 \\
\hline MABC Manual Dexterity* & 53.5 & $12 \cdot 3$ & $57 \cdot 8$ & $14 \cdot 7$ & 0.36 & 0.14 & 0.11 \\
\hline \multicolumn{8}{|l|}{ Attentional functioning } \\
\hline Orienting attentiont & 139 & 73 & 149 & 99 & 0.14 & 0.64 & 0.54 \\
\hline Alerting attention $\dagger$ & -86 & 75 & -78 & 71 & 0.12 & 0.88 & 0.83 \\
\hline Executive attention† & -174 & 210 & -253 & 215 & 0.49 & 0.06 & 0.07 \\
\hline \multicolumn{8}{|l|}{ Working memory } \\
\hline Visual forward $\dagger$ & 4.9 & 0.8 & 4.7 & 0.7 & 0.42 & 0.14 & 0.07 \\
\hline Visual backward $\dagger$ & 4.4 & 0.9 & $4 \cdot 1$ & 0.8 & 0.36 & $0 \cdot 10$ & 0.16 \\
\hline WISC-III Digit Span Forward† & $6 \cdot 4$ & 1.6 & $6 \cdot 6$ & 1.7 & 0.18 & 0.37 & 0.82 \\
\hline WISC-III Digit Span Backward† & 3.5 & 1.4 & 3.9 & $1 \cdot 3$ & 0.27 & 0.96 & 0.59 \\
\hline \multicolumn{8}{|l|}{ Information processing } \\
\hline Overall processing speed $\dagger$ & 706 & 138 & 764 & 161 & 0.35 & 0.32 & 0.28 \\
\hline \multicolumn{8}{|l|}{ Parent report of behaviour } \\
\hline CBCL Total* & $50 \cdot 0$ & $8 \cdot 6$ & $49 \cdot 7$ & $9 \cdot 3$ & $0 \cdot 10$ & 0.94 & 0.88 \\
\hline CBCL Internalising* & $49 \cdot 1$ & $7 \cdot 0$ & $50 \cdot 5$ & $9 \cdot 0$ & 0.10 & 0.57 & 0.79 \\
\hline CBCL Externalising* & $49 \cdot 1$ & $9 \cdot 8$ & 48.4 & 8.3 & 0.04 & 0.92 & 0.93 \\
\hline PDBD Inattention* & $55 \cdot 0$ & $10 \cdot 3$ & $49 \cdot 8$ & 8.7 & 0.52 & 0.39 & 0.17 \\
\hline PDBD Hyperactivity* & $53 \cdot 1$ & $10 \cdot 2$ & $50 \cdot 0$ & $6 \cdot 7$ & 0.39 & 0.52 & 0.31 \\
\hline \multicolumn{8}{|l|}{ Teacher's report of behaviour } \\
\hline TRF Total $^{\star}$ & $51 \cdot 6$ & 14.7 & $46 \cdot 6$ & $7 \cdot 3$ & 0.32 & 0.32 & 0.27 \\
\hline TRF Internalising* & 48.9 & $11 \cdot 1$ & $46 \cdot 3$ & $6 \cdot 1$ & 0.23 & 0.29 & 0.48 \\
\hline TRF Externalising ${ }^{*}$ & $50 \cdot 6$ & $13 \cdot 1$ & $46 \cdot 0$ & 5.7 & 0.38 & 0.53 & 0.25 \\
\hline TDBD Inattention ${ }^{*}$ & $58 \cdot 0$ & $16 \cdot 9$ & $52 \cdot 1$ & $9 \cdot 0$ & 0.32 & 0.65 & 0.30 \\
\hline TDBD Hyperactivity* & 54.5 & $13 \cdot 8$ & 48.9 & $7 \cdot 3$ & 0.43 & 0.32 & 0.29 \\
\hline
\end{tabular}

GA, gestational age; SES, socio-economic status; WISC-III, Wechsler Intelligence Scales for Children, 3rd edition; FSIQ, full-scale intelligence quotient; MABC, Movement Assessment Battery for Children; CBCL, Child Behaviour Checklist; PDBD, parent-rated Disruptive Behaviour Disorders questionnaire; TRF, Teacher's Report Form; TDBD, teacher version of the Disruptive Behaviour Disorders questionnaire.

${ }^{*}$ Normalised T-scores. Higher scores indicate poorer performance.

† Raw scores. 
glutamine and placebo groups. Including serious neonatal infections as a second between-subjects factor did not change the results of the primary analysis.

\section{Discussion}

The majority of long-term cognitive, motor and behavioural outcome measures were not different in the glutamine and placebo groups. These findings demonstrated that glutamine supplementation between day 3 and day 30 of life did not have beneficial or detrimental effects on a wide range of cognitive and motor functions or on parent- and teacherreported behavioural outcomes at school age. However, ball skills as measured by the MABC were found to be significantly poorer in the glutamine group as compared with the placebo group, suggesting the possibility that glutamine treatment in the first month after birth may have adverse effects on visuomotor development.

Many factors may influence developmental outcomes between birth and 8 years of age, thereby diluting the effects of enteral glutamine supplementation between day 3 and day 30 of life. The current cognitive, motor and behavioural measures were selected to maximise the ability to chart any long-term effects on outcomes at school age. Furthermore, long-term effects on cognitive, motor and behavioural development of enteral glutamine supplementation could be expected, as glutamine treatment reduced the incidence of serious neonatal infections known to affect brain development $^{(3,15)}$. Indeed, significant associations were present between the presence of serious neonatal infections and the selected behavioural outcomes, including inattention and hyperactivity, indicating that an increase in the presence of serious neonatal infections may be associated with poorer outcomes. Nonetheless, no major effects of enteral glutamine supplementation on long-term cognitive, motor and behavioural outcomes were present.

The unfavourable effect of glutamine supplementation on visuomotor development as measured with the Ball Skills scale of the MABC was an unexpected finding, as our previous work has shown no unfavourable short-term effects of enteral glutamine supplementation ${ }^{(17)}$, and no direct detrimental effects of glutamine on the brain have been reported ${ }^{(28)}$. However, derivates of glutamine, including glutamate and ammonia, have been associated with neurotoxicity ${ }^{(28,29)}$, and could underlie detrimental outcomes following glutamine treatment. In patients with hepatic encephalopathy, ammonia was shown to affect the metabolism and function of astrocytes, leading to astrocytic swelling which in turn leads to cerebral oedema. Glutamine is synthesised in excess from ammonia and glutamate by glutamine synthetase, an astrocyte enzyme. Recent data suggest that many aspects of ammonia toxicity in hepatic encephalopathy are mediated by glutamine ${ }^{(30)}$. Nevertheless, plasma concentrations of glutamine, glutamate and other amino acids were not different between the glutamine and placebo groups in the neonatal period in the present study sample ${ }^{(31)}$, and the highest median glutamate $(68 \mu \mathrm{mol} / 1$ glutamine group; $70 \mu \mathrm{mol} / 1$ placebo group) or glutamine concentrations $(555 \mu \mathrm{mol} / \mathrm{l}$ glutamine group;
$515 \mu \mathrm{mol} / 1$ placebo group) were within normal reference ranges ${ }^{(32)}$. Furthermore, if adverse neurological effects as a consequence of neurotoxicity occurred, more widespread consequences on cognitive, motor and behavioural outcomes would have been expected, given that visuomotor skills concern interplay between perception, motor skills and timing, involving multiple brain systems. Hence, direct effects on the brain seem unlikely, and we speculate that the presence of differences in visuomotor skills between the glutamine and placebo groups is related to baseline differences in visuomotor abilities at the moment of randomisation.

Several studies in VLBW infants have investigated the effects of parenteral or enteral glutamine supplementation on morbidity, mortality and (growth) outcome in the neonatal period $^{(10,18,33-36)}$. No evidence of toxicity of glutamine supplementation was found in these clinical trials, but the results of efficacy on a limited number of outcomes have been mixed $^{(12)}$, possibly due to differences in supplementation methods, dose and definition of infections. As a consequence, the use of glutamine supplementation has not become routine. However, the effects of glutamine supplementation on the incidence of serious neonatal infections warrants further investigation, given that our findings indicate no clear evidence of long-term detrimental effects on motor, cognitive and behavioural development, underlining the relative safe use of glutamine supplementation in very preterm and/or VLBW children in future research or clinical practice.

The present study has some limitations that need to be taken into account when interpreting the findings. First, the power of analyses was somewhat limited due to the fact that not all children were still participating nearly 8 years after original enrolment in the present study. However, drop-out rates were low for this type of long-term follow-up, and drop-out was equally present in the glutamine and placebo groups. Furthermore, power was sufficient to detect any medium- to large-sized beneficial or detrimental effects of glutamine treatment, as calculated using G-power software ${ }^{(37)}$. Second, to reduce the chance of a type 2 error that erroneously may lead to the conclusion that no detrimental or beneficial effects of glutamine-enriched feeding on long-term outcomes exist, no $\alpha$ correction for multiple comparisons was conducted. The flip side of the coin is that our finding of unfavourable effects on visuomotor abilities of glutamine intervention might result from an unadjusted $\alpha$ level in our statistical tests.

In summary, with one single exception (visuomotor abilities), no medium- or large-sized beneficial or detrimental effects of short-term enteral glutamine supplementation in the neonatal period were found on long-term cognitive, motor and behavioural outcomes of very preterm and/or VLBW children at school age.

\section{Acknowledgements}

The present study was supported by an unrestricted research grant from Danone ${ }^{\circledR}$ (Friederichsdorf, Germany). Danone ${ }^{\circledR}$ also supported manufacture of the glutamine supplement used in the present study but had no involvement in the analysis of the data or the interpretation of the results of this 
investigation. J. F. d. K. carried out the practical work, the analyses and wrote the manuscript. In addition, A. v. Z., G. B. and $\mathrm{H}$. N. L. co-wrote the manuscript and gave advice. J. O. and R. M. V. E. co-wrote the manuscript, gave advice and supervised the project. J. F. d. K., J. O., A. v. Z., H. N. L. and R. M. v. E. have no conflict of interest. G. B. is an employee of Danone Research, Centre for Specialised Nutrition.

\section{References}

1. de Kieviet JF, Piek JP, Aarnoudse-Moens CS, et al. (2009) Motor development in very preterm and very low-birthweight children from birth to adolescence: a meta-analysis. JAMA 302, 2235-2242.

2. Bhutta AT, Cleves MA, Casey PH, et al. (2002) Cognitive and behavioral outcomes of school-aged children who were born preterm: a meta-analysis. JAMA 288, 728-737.

3. Adams-Chapman I \& Stoll BJ (2006) Neonatal infection and long-term neurodevelopmental outcome in the preterm infant. Curr Opin Infect Dis 19, 290-297.

4. Neu J \& Li N (2007) Pathophysiology of glutamine and glutamate metabolism in premature infants. Curr Opin Clin Nutr Metab Care 10, 75-79.

5. Khan J, Iiboshi Y, Cui L, et al. (1999) Alanyl-glutaminesupplemented parenteral nutrition increases luminal mucus gel and decreases permeability in the rat small intestine. JPEN J Parenter Enteral Nutr 23, 24-31.

6. Rhoads JM, Argenzio RA, Chen W, et al. (1997) L-Glutamine stimulates intestinal cell proliferation and activates mitogenactivated protein kinases. Am J Physiol 272, G943-G953.

7. Fox AD, Kripke SA, De Paula J, et al. (1988) Effect of a glutamine-supplemented enteral diet on methotrexate-induced enterocolitis. JPEN J Parenter Enteral Nutr 12, 325-331.

8. Salvalaggio PR \& Campos AC (2002) Bacterial translocation and glutamine. Nutrition 18, 435-437.

9. Souba WW, Klimberg VS, Hautamaki RD, et al. (1990) Oral glutamine reduces bacterial translocation following abdominal radiation. $J$ Surg Res $\mathbf{4 8}, 1-5$.

10. Neu J, Roig JC, Meetze WH, et al. (1997) Enteral glutamine supplementation for very low birth weight infants decreases morbidity. J Pediatr 131, 691-699.

11. van den Berg A, van Elburg RM, Westerbeek EA, et al. (2005) Glutamine-enriched enteral nutrition in very-lowbirth-weight infants and effects on feeding tolerance and infectious morbidity: a randomized controlled trial. $A m J$ Clin Nutr 81, 1397-1404.

12. Tubman TR, Thompson SW \& McGuire W (2008) Glutamine supplementation to prevent morbidity and mortality in preterm infants. The Cochrane Database of Systematic Reviews issue 1, CD001457. http://www.mrw.interscience. wiley.com/cochrane/clsysrev/articles/CD001457/frame.html

13. Kermorvant-Duchemin E, Laborie S, Rabilloud $\mathrm{M}$, et al. (2008) Outcome and prognostic factors in neonates with septic shock. Pediatr Crit Care Med 9, 186-191.

14. Schlapbach LJ, Aebischer M, Adams M, et al. (2011) Impact of sepsis on neurodevelopmental outcome in a Swiss National Cohort of extremely premature infants. Pediatrics 128, e $348-\mathrm{e} 357$.

15. Stoll BJ, Hansen NI, Adams-Chapman I, et al. (2004) Neurodevelopmental and growth impairment among extremely low-birth-weight infants with neonatal infection. JAMA 292, 2357-2365.

16. Volpe JJ (2008) Postnatal sepsis, necrotizing entercolitis, and the critical role of systemic inflammation in white matter injury in premature infants. $J$ Pediatr 153, 160-163.
17. van Zwol A, van den Berg A, Huisman J, et al. (2008) Neurodevelopmental outcomes of very low-birth-weight infants after enteral glutamine supplementation in the neonatal period. Acta Paediatr 97, 562-567.

18. Korkmaz A, Yurdakok M, Yigit S, et al. (2007) Long-term enteral glutamine supplementation in very low birth weight infants: effects on growth parameters. Turk J Pediatr 49, 37-44.

19. Wechsler D (2002) WISC-III Handleiding (WISC-III Manual). London: The Psychological Corporation.

20. Groth-Marnat G (1997) Handbook of Psychological Assessment, 3rd ed. New York: Wiley.

21. Nutley SB, Söderqvist S, Bryde S, et al. (2010) Measuring working memory capacity with greater precision in the lower capacity ranges. Dev Neuropsychol 35, 81-95.

22. Henderson SE \& Sugden DA (1992) Movement Assessment Battery for Children: Manual. London: The Psychological Corporation.

23. Fan J, McCandliss BD, Fossella J, et al. (2005) The activation of attentional networks. Neuroimage 26, 471-479.

24. Verhulst FC, van der Ende J \& Koot HM (1996) Handleiding voor de CBCL/4-18 (Manual for the CBCL/4-18). Rotterdam: Erasmus University, Department of Child and Adolescent Psychiatry, Sophia Children's Hospital.

25. Oosterlaan J, Scheres A, Antrop I, et al. (2000) Vragenlijst voor Gedragsproblemen bij Kinderen (VvGK). Nederlandse bewerking van de Disruptive Behavior Disorders Rating Scale (Dutch Translation of the Disruptive Behavior Disorders Rating Scale). Lisse, The Netherlands: Swets \& Zeitlinger.

26. Verhulst FC, van der Ende J \& Koot HM (1997) Handleiding voor de Teacher's Report Form (TRF) (Manual for the Teacher's Report Form (TRF)). Rotterdam: Erasmus University.

27. Cohen J (1988) Statistical Power Analyses for the Behavioral Sciences, 2nd ed. Hillsdale, NY: Erlbaum.

28. Garlick PJ (2001) Assessment of the safety of glutamine and other amino acids. J Nutr 131, Suppl. 9, 2556S-2561S.

29. Suárez I, Bodega G \& Fernández B (2002) Glutamine synthetase in brain: effect of ammonia. Neurochem Int 41, 123-142.

30. Albrecht J, Zielinska M \& Norenberg MD (2010) Glutamine as a mediator of ammonia neurotoxicity: a critical appraisal. Biochem Pharmacol 80, 1303-1308.

31. van den Berg A, van Elburg RM, Teerlink T, et al. (2005) A randomized controlled trial of enteral glutamine supplementation in very low birth weight infants: plasma amino acid concentrations. J Pediatr Gastroenterol Nutr 41, 66-71.

32. Oladipo OO, Weindel AL, Saunders AN, et al. (2011) Impact of premature birth and critical illness on neonatal range of plasma amino acid concentrations determined by LC-MS/ MS. Mol Genet Metab 104, 476-479.

33. Bober-Olesińska K \& Kornacka MK (2005) Effects of glutamine supplemented parenteral nutrition on the incidence of necrotizing enterocolitis, nosocomial sepsis and length of hospital stay in very low birth weight infants (article in Polish). Med Wieku Rozwoj 9, 325-333.

34. Poindexter BB, Ehrenkranz RA, Stoll BJ, et al. (2004) Parenteral glutamine supplementation does not reduce the risk of mortality or late-onset sepsis in extremely low birth weight infants. Pediatrics 113, 1209-1215.

35. Thompson SW, McClure BG \& Tubman TR (2003) A randomized, controlled trial of parenteral glutamine in ill, very low birthweight neonates. J Pediatr Gastroenterol Nutr 37, 550-553.

36. Vaughn P, Thomas P, Clark R, et al. (2003) Enteral glutamine supplementation and morbidity in low birth weight infants. J Pediatr 6, 662-668.

37. Faul F, Erdfelder E, Lang AG, et al. (2007) G*Power 3: a flexible statistical power analysis program for the social, behavioral, and biomedical sciences. Behav Res Methods 39, 175-191. 